\title{
Advanced Light Wire Functional (ALF) Appliance - An Aid to Approach Growth Correction via Nature: Review Article
}

\author{
Nandakishore Rajgopal ${ }^{1}$, Anil Kumar ${ }^{2}$ \\ ${ }^{1}$ Postgraduate, Department of Orthodontics and Dentofacial Orthopaedics, AJ Institute of dental Science and Technology \\ Corresponding Author Email id: nandaku007[at]yahoo.com \\ ${ }^{2}$ Reader, Department of Orthodontics and Dentofacial Orthopaedics, AJ Institute of dental Science and Technology
}

\begin{abstract}
The review article is done in order to highlight the Advanced light wire functional (ALF) appliance in the field of orthodontics and to acknowledge its strong connection in the correction of skeletal and dental defects via the correction of cranial lesions.
\end{abstract}

Keywords: Advanced light wire functional appliance, Cranial Lesions, Biotensegrity, Osteogenesis

\section{Introduction}

In the field of orthodontics, the force is the medicine which the orthodontist prescribes for the treatment of malocclusions. As Hippocrates stated "Natural forces within us are the true healers of disease" was never thought to be fruitful until a new paradigm shift and approach was brought in dentistry with the introduction of Advanced Light Force (ALF) Appliance by Dr. Darick Nordstorm in 1982. This innovation promoted better functions such as deglutition, speech, nasal respiration and sleep by combining cranial mobility with palate, tooth and jaw alignment. There is a tremendous change in neurological regulations occurring through the cranial nerves and the autonomic nervous system due to the light force stimuli delivered in the oral cavity with the help of ALF appliance. The appliance also seems to solve or improve unrelated health issues such as TMJ disorders, postural abnormalities, sleep disorders, orofacial pain disorders etc.

\section{The Philosophy}

The three main purposes of ALF appliance is

1) To bring cranial bones into good functional motion or balance motion.

2) To develop the dental arches to align the teeth and the jaw functions together and to provide adequate space for the tongue.

3) To integrate all the above said factors to promote proper function of swallowing, speech and deglutition.

\section{Cranial movements}

Many osteopaths have concluded that there are flexion and extension movements happening in the cranial bones, the posterior part of the occiput or the squamous portion flares downwards in contradiction to the rise of basi-occiput, a rotational movement takes place in the overall sphenoid bone, this a classical example of flexion movement of the bones in the cranium, similarly there is an extension movement happening in the cranial bones after the completion of the flexion as the opposite takes place with the basi-occiput and basi-shpenoid gets projected downwards. The cranial flexion and extensions happen eight to 12 times per minute approximately. When the flexion happens the skull shortens anteroposteriorly and laterally it widens. In extension, the opposite takes place.
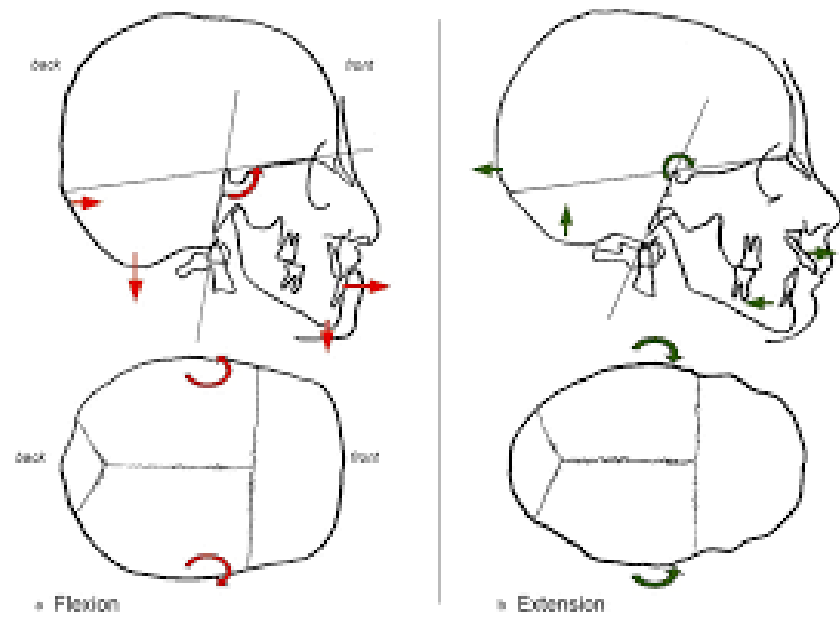

Figure 1: Sagittal and superior visualization of flexion and extension of cranial bones, and their consequences on dental

class and cranial form. (A) Flexion of Spheno-occipital synchondrosis and other bones tends towards dental class III, prognathism and long face; (B) Extension of Sphenooccipital synchondrosis and other bones tend towards dental class II, retrognathism and diminution of face length

The sphenoidal movement is transmitted throughout the whole spheno-maxillary complex, the movement of the pterygoid process downwards and upwards during flexion affects the palate- maxillary complex, resulting in widening and flattening of the palate. This could be followed with contraction and elevation of plate during the extension movement. There is a meticulous study by Baker in which he found out that the maxillary arch expands permanently as a result of cranial adjustments, Also the movement of the temporal bone around an axis which extends from external auditory meatus to the medial tip of the petrous part of the temporal bone. It carries the gleniod fossa slightly posteromedially in flexion and the squamous part of the 
temporal bone flaring anterolaterally, In extension the fossa is carried slightly anterolaterally.
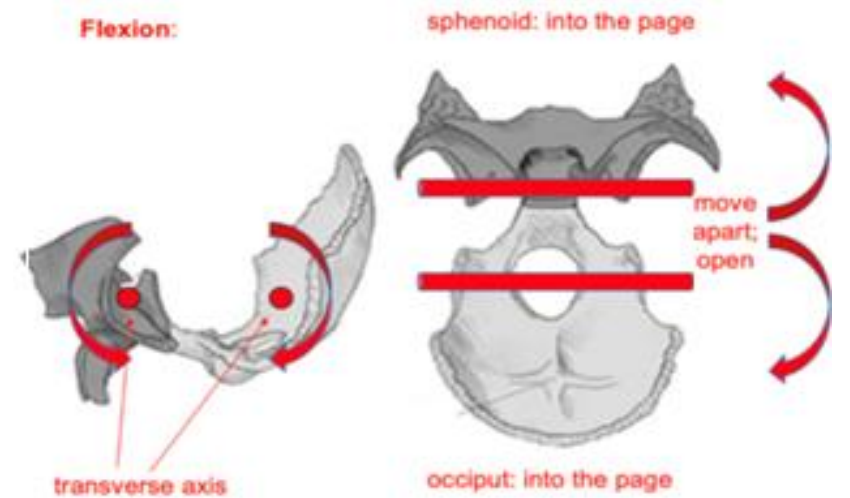

Figure 2: Flexion Cranial Somatic Dysfunction Bone Motion during Flexion

When a TMJ disorder is assessed, one must consider that the glenoid fossa is a flexible mobile structure under the influence of the temporal bone and to its orientation to the cranial mechanism ${ }^{1}$.

Correlation of cranial distortion and the ALF Philosophy The six year studies of literature and clinical material by Dr. Gavin and Dr. Strokon, they have identified that each cranial distortion predisposes to a specific malocclusion. They conclude that the cranial lesion is usually the primary etiological factor underlying different malocclusion or the cranial compensation which occur subsequent to the TMJ internal derangement which causes the dental imbalance. A diagnosis of cranial lesion should be done by the method of palpation by a trained osteopath which should also combine with radiographic evidence and articulated study models. The treatment approach should primarily be concerned to correct the cranial lesion, then the correction of mandible and to the maxilla. Dr. Gavin and Dr. Strokon advocate the correction of the cranial lesions with an approach of combining cranial adjustment by an osteopath and with light orthopaedic-type forces delivered by suitable orthodontic appliances, with their experience they mention that the force levels presently used in orthodontics can restrain the cranial rhythm in often ${ }^{1}$.

\section{The importance of Biotensegrity}

The brainstem is positioned at the back of the throat, every bite can bring a feedback message to the brain, and an output is generated from the brainstem in reference to the previous interpretations. An underlying neurobiological issue could be the culprit of the behaviours such as hyperactivity, poor attention, sensory processing, gut dysfunction, sleep disorders, balance and motor control etc. If an alternative oral input provided, an organising output may occur from brain to bite, body and behaviour is the key act provided by the use of ALF appliance on the palate with the principles of engineering design to balance stress and tension in the cranio-mandibular system termed as biotensegrity.

Experiment in rats involving orthodontic wire expansion have showed secondary cartilage to undergo chondrogenic and osteogenic differentiation in the maxillary arch, this concludes that mechanical stimulus in the natural cycle of growth can cause reorganization in bone and cartilage ${ }^{2}$.

\section{Parts of ALF appliance}

The ALF consist of a 0.025 Elgiloywire which is very flexible and resilient in nature. It also consist of a 0.025 Elgiloy lingual wire that extends around the lingual surface of molar to opposite side molar lingual aspect, Omega loops are incorporated into the arch wire where expansion or spacing is desired. Crescents are soldered to the wire for retention purpose in the premaxillary region. Wire cribs create retention on the molars and can be designed for specific molar movement. Bonded composites can be used on various teeth to provide extra retention. The standard ALF touches only four teeth and it promotes spaces between all the teeth. Use of finger springs in the distal end of the wire to distalize second molars could also be carried out Individual loops can be made to facilitate specific molar movement. In fact the ALF is a combination of many appliances constructed in a single appliance ${ }^{3}$.

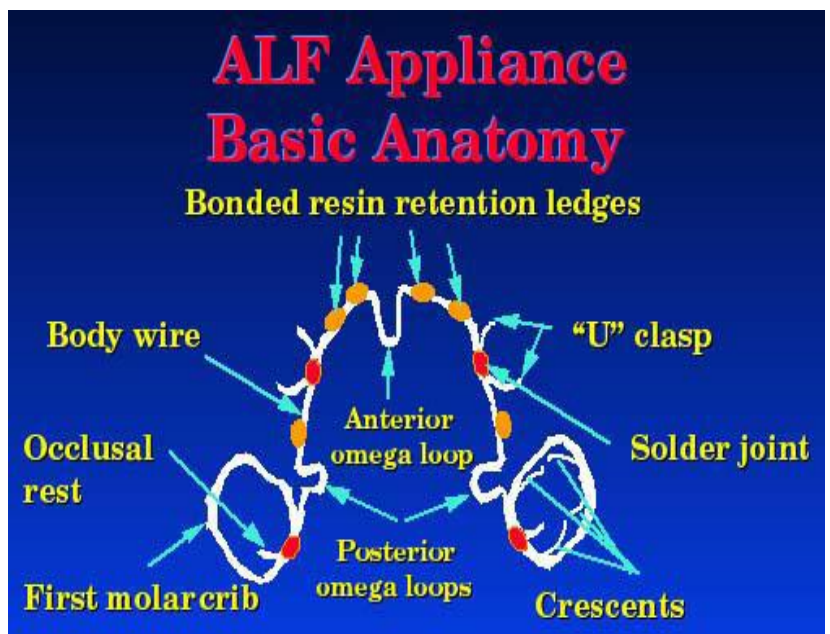

Figure 3: Parts of the ALF Appliance

\section{Working of the ALF}

The gentle sensory input provided by the ALF can alter osteogenesis, relieving the cranial stress pattern and promote proper transverse development of jaws, as the cranial nerve sensory input changes, a spontaneous corrective motor output follows, autonomic and neuro- hormonal balances, as the sympathetic and parasympathetic rebalancing happens proper internal function begin. In adjunct to ALF therapy, proper tongue exercise are followed makes the treatment more efficient by correcting the position of the tongue ${ }^{2}$. ALF also exerts a lateral force throughout the treatment, which are interrupted by the tooth proprioceptors and periodontal fibres just as the chewing force, in children it creates spaces for eruption of teeth through appositional bone and cartilaginous growth, in adults it is promoted by appositional growth. Overall the ALF not only transmit the gentle forces to the palate but also subsequently transmits force to the brainstem which houses the pituitary gland. The biomimetic tongue function of the ALF is also to be considered giving stability to the cranio-facial complex. Dr. James and Dr. Strokon stated that the ALF appliance primarily assist in the correction of cranial 'lesion' and only secondarily to move teeth, but this can be further interpreted as the cranial

Volume 10 Issue 6, June 2021 
distortion and tooth arrangement issues will occur in 'hand in hand'

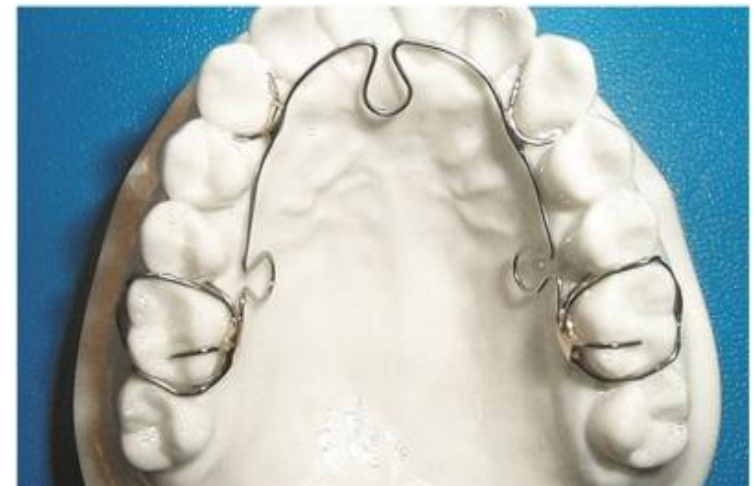

Figure 4: Image of the ALF Appliance design

\section{Conclusion}

The ALF appliance therapy helps to return the normal balanced biotensegrity system in the cranio-mandibular complex. The correction of oral rest postural position promotes corrective orofacial form and its function, the cranial nerve and autonomic regulations which help the cellular, biochemical, hormonal, neurological and behavioural organization ${ }^{1}$. The use of light elgiloy wire to generate gentle consistent force and removable in nature promotes adult patient compliance as well as efficiency of the appliance ${ }^{3}$. The ALF therapy integrates the field of orthodontics with osteopathy and is potent to open a new frame shift to the existing paradigm. Despite ALF was introduced in the year of 1982 by Dr. Nordstorm the controversy exist that this evidence based therapy is not rightly looked upon the way it has to be or maybe not given the preference or ignored in the treatment mechanics for the desired patients where this appliance can fetch fruitful results when used alone or in adjunct with other orthodontic appliances.

\section{References}

[1] James GA, Strokon D. The significance of cranial factors in diagnosis and treatment with the advanced lightwire functional appliance. International Journal of Orthodontics-Milwaukee-. 2003 Jan 1; 14(3):17-

[2] Bronson JM, Bronson JA. Introducing the Advanced Light Force (ALF) Appliance. Oral Health. 2015 Mar $1: 10-7$.

[3] Delz E. The ALF (Advanced Lightwire Functional Appliance) Creating Facial Beauty and Balance.IJO. 2009;20(2).

[4] Bronson JM, Bronson JA. Early treatment with the ALF functional appliance.IJO. 2014;25(1)

[5] Smith GH, Ashton H. Alternative lightwire functionals (ALF). Funct Orthod.1995; 12(4):35-38.

[6] Ranly DM. A synopsis of craniofacial growth. Appleton \& Lange; 1988.

[7] Walker R. Chirodontics. A Treatment Paradigm for the New Millennium. The Functional orthodontist. 1998;15(4):12-5.

[8] James GA, Strokon D. Cranial strains and malocclusion VII: a review. IJO. 2006; 17(4).
[9] James G, Strokon D. Cranial strains and malocclusion VIII: palatal expansion. International journal of orthodontics (Milwaukee, Wis.). 2009 Jan 1; 20(4):1530 .

Volume 10 Issue 6, June 2021 www.ijsr.net 